\title{
Ferramentas Avaliativas Disponíveis em um Ambiente Virtual de Aprendizagem usada no Planejamento de um curso através do Mapa de Dependências
}

\author{
Roberto Douglas da Costa - IFRN - douglas.costa@ifrn.edu.br \\ Rommel Wladimir de Lima - UERN - rommelwladimir@uern.br \\ Thiago Reis da Silva - UFRN - trsilva.si@gmail.com \\ Selma Marcia Pontes Teixeira da Rocha - UERN/UFERSA - \\ selmapontes@yahoo.com.br \\ Dimas Kastiberg Fernandes - UERN/UFERSA - k45t1b@gmail.com
}

Resumo: Este artigo apresenta uma classificação cognitiva, através da taxonomia de Bloom, para as atividades avaliativas dispostas nos Ambientes Virtuais de Aprendizagem. Para isso foi usado um algoritmo para realizar um levantamento dos verbos que aparecem na descrição de todas as atividades utilizadas em cursos e ou disciplinas ministradas através dos Ambientes Virtuais de Aprendizagem Moodle em uma instituição de ensino que trabalha com cursos na modalidade à distância $e$ relaciona-los aos verbos referentes a cada classe do domínio cognitivo da Taxonomia de Bloom, alcançando assim o objetivo deste trabalho.

Palavras-chaves: Ambiente Virtual de Aprendizagem, Planejamento, Mapa de Dependência, Moodle.

\section{Tools Available evaluative in a Virtual Learning Environment used in planning a course through the Dependency Map}

Abstract: This paper presents a cognitive classification through of Bloom's taxonomy for the evaluation activities arranged in Virtual Learning Environments. For this we used an algorithm to conduct a survey of the verbs that appear in the description of all activities and used in courses or courses taught through the Moodle Virtual Learning Environments in an educational institution that works with courses in distance mode and relates them to verbs for each class of the cognitive domain of Bloom's Taxonomy, thus achieving the objective of this work.

Keywords: Virtual Learning Environments, Planning, Dependency Map, Moodle.

\section{Introdução}

Hoje com a crescente demanda dos cursos oferecidos pela modalidade de Ensino a Distância (EaD) juntamente como as novas Tecnologias da Informação e Comunicação (TIC) [Coelho Neto et. al. 2011] desenvolvidas, surge uma nova modalidade de curso a distância através da Internet, que são os cursos online.

Foi através deste cenário que os Ambientes Virtuais de Aprendizagem (AVA) [Gluz, Galafasi e Penteado 2011], tomaram forma e foram desenvolvidos para gerir essa nova modalidade de apoio ao desenvolvimento e andamento de cursos.

Esses ambientes, recheados de recursos pedagógicos, têm o intuito auxiliar o professor no processo de criação, acesso, planejamento, controle e desenvolvimento de curso ou disciplina, para ser executado num meio onde a interação professor-aluno 
possa acontecer à distância, isto é, em ambientes fisicamente distintos [Piva Júnior, Pupo e Gamez 2011].

Para isso, a organização didática e pedagógica terá de ser planejada de modo que os alunos assumam a construção autônoma do seu processo de aprendizagem sem a presença física do professor. Uma forma de se obter essa autonomia será através dos Objetivos Educacionais [Silva et. al., 2012] definidos pelo professor durante o planejamento do curso ou disciplina [Lima 2009].

Os objetivos educacionais são metas ou comportamentos esperados a serem atingidas, durante o transcorrer de um curso ou disciplina, na qual é obedecida uma hierarquia cognitiva onde o aluno só consegue alcançar o domínio definido pelo professor para conclusão do seu curso ou disciplina, se detiver as capacidades ou habilidades inferiores.

Uma forma de se trabalhar com objetivos educacionais é através da Taxionomia de Bloom, proposta por Benjamin Bloom na década de 50 que juntamente com seus colaboradores, M. D. Englehart, E. J. Furst, W. H. Hill e D. Krathwohl criaram uma força tarefa para discutir, definir e criar uma taxonomia dos objetivos educacionais dividindo o trabalho de acordo com o domínio específico de desenvolvimento que pode ser cognitivo, afetivo e psicomotor [Ferraz e Belhot 2010 apud Lomena 2006].

No desenvolvimento deste trabalho daremos ênfase ao domínio cognitivo, que abrange a aprendizagem intelectual do aluno. Nesse domínio os objetivos educacionais estão divididos em seis classificações (classes) que são: Avaliação, Síntese, Análise, Aplicação, Compreensão e Conhecimento.

A Taxionomia de Bloom permite associar os objetivos educacionais ao planejamento e a avaliação do aluno, mas no caso do aluno não conseguir atingir o objetivo desejado, ela não define os mecanismos necessários para se identificar onde está o problema. O que pode restringir a autonomia do aluno, característica necessária à EaD.

Como alternativa para sanar esse problema foi desenvolvido o Mapa de Dependências [Lima e Fialho 2008]. Ele permite ao professor definir, além do objetivo educacional a ser alcançado, os comportamentos necessários para se atingir esse objetivo. Assim, o Mapa de Dependências (MD) é uma ferramenta constituída de um conjunto de objetivos educacionais relacionados entre si, usando a hierarquia definida na Taxionomia de Bloom [Lima 2009].

Assim, o MD apresenta a relação de dependência do objetivo educacional pretendido com os objetivos educacionais das classes inferiores como forma de contribuição ao alcance do objetivo educacional final. Para isso é preciso que se identifique qual ou quais os comportamentos anteriores do aluno influenciam no alcance do objetivo educacional proposto.

Todo esse relacionamento de dependência é gerado através do MD e essa sequencia de passos se repete até que os comportamentos esperados dos alunos atinjam o nível mais baixo da taxionomia, ou até um nível onde o professor ache que não precisa mais descer.

Porém, o MD trabalha apenas com objetivos educacionais no nível de planejamento, mas não define os elementos avaliativos que possibilite a identificação do alcance desses objetivos. 
Devido a isso, este trabalho teve como propósito inserir o processo de avaliação no MD através da criação de um ambiente que possa, utilizando as ferramentas avaliativas já desenvolvidas em um AVA, classificar de forma cognitiva cada uma dessas atividades e associá-las aos objetivos educacionais definidos no MD, durante o planejamento do curso ou disciplina, permitindo ao professor, não só apenas planejar o desenvolvimento da disciplina, mas também escolher que atividades avaliativas poderá usar para que o aluno possa atingir o objetivo educacional por ele definido.

Para demonstrar melhor este trabalho, além desta introdução - Seção 1 - este artigo foi dividido em mais sete Seções, onde na Seção 2 apresentaremos o Ambiente Virtual de Aprendizagem e suas atividades avaliativas, a Seção 3 aborda sobre os Objetivos Educacionais e a Taxionomia de Bloom. Na Seção 4 veremos as atividades avaliativas através da ferramenta Mapa de Dependência, consequentemente na Seção 5 será apresentada a Metodologia. Na Seção 6 será demonstrada a Infraestrutura utilizada e, por fim, na Seção 7 serão expostas as considerações finais.

\section{Ambiente Virtual de Aprendizagem e suas atividades avaliativas}

O AVA usado nesta pesquisa foi o Moodle que é um acrônimo para "Modular ObjectOriented Dynamic Learning Environment” [Silva 2011] que em português poderia ser traduzido como "Ambiente de aprendizado dinâmico e modular orientado a objetos".

Neste AVA existe um bloco de atividades onde o professor, através do campo descrição, descreve a seus alunos, usando verbos no imperativo, o que ele deve desenvolver durante a realização de uma determinada tarefa, permitindo assim uma maior interatividade e interação entre os participantes [Costa et. al., 2012].

Essas ferramentas podem ser divididas em atividades síncronas e atividades assíncronas. A comunicação síncrona acontece quando os comunicadores estão conectados no ambiente ao mesmo tempo e a interação acontece em tempo real. Já a comunicação assíncrona não necessita da presença física dos participantes, eles podem se comunicar sem as limitações impostas pelo tempo.

Entre as ferramentas avaliativas analisadas neste trabalho estão as seguintes:

- Síncronas: Chat;

- Assíncronas: Fórum, Escolha, Glossário, Diário, Questionário, tarefa - texto online, tarefa - envio de arquivo único, tarefa - modalidade avançada de carregamento de arquivos, tarefa - atividade off-line, Wiki, Lição, Base de Dados e Pesquisa de avaliação.

Embora os AVA disponibilizem um grande conjunto de ferramentas avaliativas, a forma de quando e como utilizar essas ferramentas não está bem definido. Nesse sentido, a próxima seção apresenta um estudo quantitativo do uso das atividades avaliativas usadas no AVA Moodle e o domínio cognitivo da Taxonomia de Bloom.

\section{Objetivos Educacionais e a Taxonomia de Bloom}

Segundo Bloom et. al., (1977) os objetivos educacionais, são maneiras ou modos de como os alunos modificam seus pensamentos, seus sentimentos e suas ações. A Taxonomia de Bloom dividiu os objetivos educacionais em três domínios: cognitivo, afetivo e psicomotor.

Portanto, este trabalho foi abordado apenas o domínio cognitivo da Taxonomia de Bloom que representa uma classificação estruturada em níveis de dificuldade 
crescente que vai do entendimento mais simples ao mais complexo, onde o aluno para desenvolver uma certa habilidade de nível mais alto deverá ter o domínio de habilidades de níveis inferiores a qual ele deseja atingir [Lima 2009].

Como apresentado na Tabela 1, a classificação hierárquica dos processos cognitivos está dividida em seis níveis de complexidade, que são: Avaliação, Síntese, Análise, Aplicação, Compreensão e Conhecimento. Para cada categoria desta Taxonomia é exibida uma lista de verbos imperativos relacionados que são usados como suporte ao planejamento sistêmico dos cursos e ou disciplinas na estruturação dos seus objetivos, estratégias e avaliações.

Tabela 1 - Estruturação da Taxonomia de Bloom do domínio Cognitivo. Fonte: [Costa et. al., 2012].

\begin{tabular}{|c|c|}
\hline Categoria & Descrição \\
\hline \multirow{2}{*}{ 1. Conhecimento } & $\begin{array}{l}\text { Definição: O aluno irá recordar ou reconhecer informações, ideias, } \\
\text { e princípios na forma (aproximada) em que foram aprendidos. }\end{array}$ \\
\hline & $\begin{array}{l}\text { Verbos: escreva, liste, rotule, mostre, tabule, enumere, copie, } \\
\text { selecione, nomeie, diga, defina, reproduza, relate, identifique, cite, } \\
\text { colete, evoque. }\end{array}$ \\
\hline \multirow{2}{*}{ 2. Compreensão } & $\begin{array}{l}\text { Definição: O aluno traduz, compreende ou interpreta informação } \\
\text { com base em conhecimento prévio. }\end{array}$ \\
\hline & $\begin{array}{l}\text { Verbos: explique, associe, deslíngua, estenda, estimule, agrupe, } \\
\text { sumarize, converta, discuta, traduza, ordene, diferencie, resuma, } \\
\text { parafraseie, descreva, interprete, ilustre. }\end{array}$ \\
\hline \multirow{2}{*}{ 3. Aplicação } & $\begin{array}{l}\text { Definição: O aluno seleciona, transfere, e usa dados e princípios } \\
\text { para completa um Problema ou tarefa com um mínimo de } \\
\text { supervisão. }\end{array}$ \\
\hline & $\begin{array}{l}\text { Verbos: use, compute, resolva, aplique, calcule, termine, } \\
\text { experimente, demonstre, descubra, determine, torne, estabeleça, } \\
\text { articule, transfira, ensine, prepare, construa. }\end{array}$ \\
\hline \multirow{2}{*}{ 4. Análise } & $\begin{array}{l}\text { Definição O aluno distingue, classifica, e relaciona pressupostos, } \\
\text { hipóteses, evidências ou estruturas de uma declaração ou questão. }\end{array}$ \\
\hline & $\begin{array}{l}\text { Verbos: analise, classifique, categorize, compare, contraste, } \\
\text { deduza, arranje, conecte, divida, priorize, indique, diagrame, } \\
\text { discrimine, focalize, separe. }\end{array}$ \\
\hline \multirow{2}{*}{ 5. Síntese } & $\begin{array}{l}\text { Definição: O aluno cria, integra e combina ideias num produto, } \\
\text { plano ou proposta, novos para ele. }\end{array}$ \\
\hline & $\begin{array}{l}\text { Verbos: crie, proponha, formule, modifique, planeje, elabore, } \\
\text { hipótese, invente, projete, desenvolva, ligue, componha, generalize, } \\
\text { substitua, integre, rearranje, reescreva, adapte, antecipe, compile. }\end{array}$ \\
\hline \multirow[t]{2}{*}{ 6. Avaliação } & $\begin{array}{l}\text { Definição: O aluno aprecia, avalia ou critica com base em padrões } \\
\text { e critérios específicos. }\end{array}$ \\
\hline & $\begin{array}{l}\text { Verbos: julgue, argumente, avalie, recomende, critique, justifique, } \\
\text { decida, teste, convença, conclua. }\end{array}$ \\
\hline
\end{tabular}

\section{Trabalhando as atividades avaliativas através da ferramenta Mapa de Dependência}

A avaliação é um processo que aprecia e diagnóstica soluções dadas a um problema proposto. Um modelo padrão de avaliação deve agregar, em um mesmo AVA, a avaliação informal com a formal, objetivando sempre a identificação dos problemas que podem surgir na aprendizagem do aluno durante o transcorrer do curso ou disciplina. 
Este modelo deverá fazer uso da avaliação formativa com o propósito de prover resultados que gerem mudanças na metodologia de ensino usada no ambiente com objetivo de melhorar a aprendizagem desses alunos.

As atividades avaliativas dispostas em AVA têm como objetivo quantificar ou qualificar informações descritas pelo aluno no processo de tomada de decisão. Este ato consiste em verificar se os objetivos exigidos para solução de um problema estão logrando êxito e em que medida estes objetivos estão sendo atingidos no transcorrer do processo aprendizagem e construção do conhecimento do aluno.

O MD - ilustrado na Figura 1 - possibilita planejar um curso virtual através de objetivos educacionais. Para isso, ele permite relacionar as dependências existentes entre o objetivo educacional pretendido, isso quer dizer, até onde se quer que o aluno chegue ao aprendizado, com os objetivos educacionais necessários para se atingi-lo.

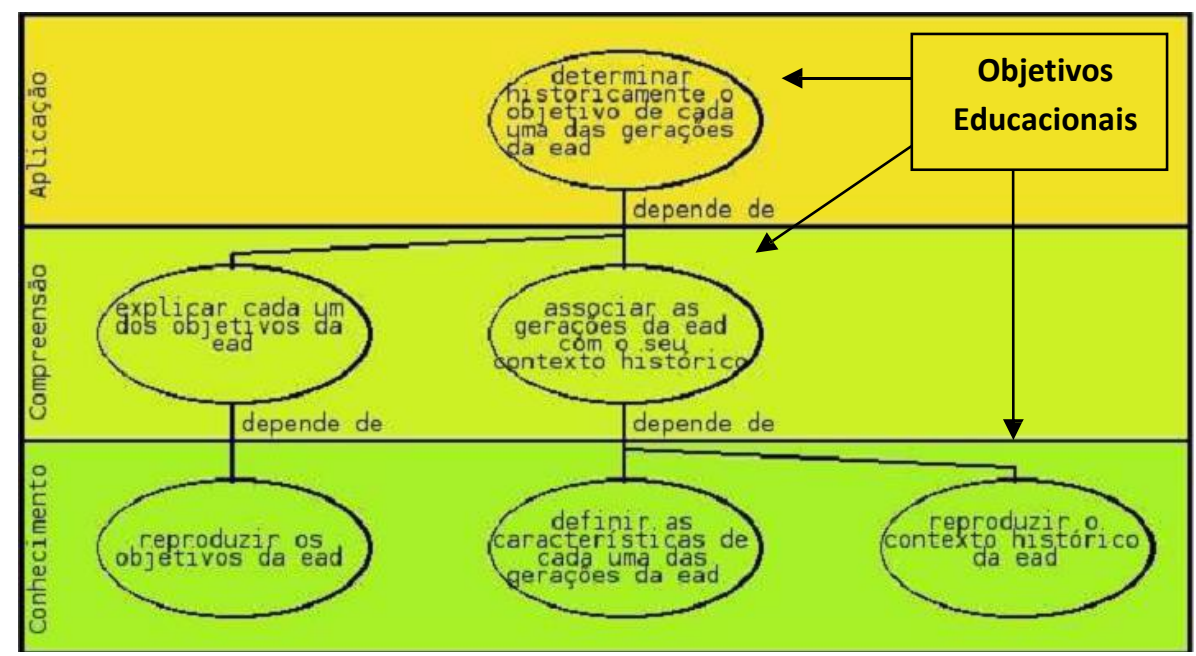

Figura 1 - Exemplo de MD. Fonte: Adaptado de [Lima 2009].

Contudo falta a forma de como avaliar o grau de conhecimento já desenvolvido pelo aluno e sua evolução objetivando o objetivo educacional final. Devido a isso, este trabalho apresenta a inserção do processo de avaliativo, através das atividades avaliativas já desenvolvidas em uma AVA.

Para isso foi feita a classificação cognitiva de todas as atividades avaliativas do AVA em estudo e as relacionou com cada objetivo educacional definido durante o planejamento do curso ou disciplina, através da ferramenta MD.

\section{Metodologia}

As atividades acadêmicas desenvolvidas foram divididas em cinco fases. Na primeira fase faremos uma revisão literária sobre as ferramentas avaliativas existentes nos AVA e sua relação com os objetivos educacionais definidos através do domínio cognitivo da Taxionomia de Bloom. Logo depois fizemos a implementação da ferramenta, seguida pelas fases de testes e avaliação da ferramenta proposta.

Na revisão literária foram levantados os aspectos relacionados a trabalhos já desenvolvidos na área em estudo, como também os conceitos relacionados com o trabalho.

Na segunda fase foi feita uma análise quantitativa dos verbos usados na descrição de todas as atividades avaliativas usados pelo Moodle e associo-as a uma ou mais classes do domínio cognitivo pertencente à Taxionomia de Bloom, onde 
conseguimos, através da execução do algoritmo exibido na Tabela 2, encontrar a quantidade de verbos associados a cada atividade explorada.

Tabela 2 - Algoritmo usado para recuperar os verbos usados em atividades.

\section{Início}

2. Para cada tipo de atividade faça;

3. Para cada verbo da tabela de verbo da taxonomia de Bloom faça;

4. Busque o verbo da tabela de verbos da taxonomia de Bloom, na descrição de

5. cada atividade;

6. $\quad$ Se verbo encontrado faça;

$7 \quad$ Armazene $o$ verbo e o tipo de atividade;

8. $\quad$ Fim do se;

$9 \quad$ Fim do para;

10. Fim do para;

11. Para cada tipo de atividade faça;

12. Imprima tipo de atividade atual;

13. Para cada verbo da tabela de verbo da taxonomia de Bloom faça;

14. Imprima a quantidade de verbos relacionados a cada tipo de atividade, e o nome do verbo;

15. Fim do para;

16. Fim do para;

17. Fim.

De posse destas informações foi possível identificar os verbos mais utilizados (verbos fortes) nas descrições de cada atividade do AVA em estudo. Através da associação dos verbos representativos das classes cognitivas da Taxonomia de Bloom, descritos na Tabela 1, com os verbos representativos de cada atividade do AVA Moodle, foi possível alcançar uma classificação cognitiva para cada atividade avaliativa do AVA trabalhado, como mostra a Figura 2.

\begin{tabular}{|c|c|}
\hline \multirow{2}{*}{$\begin{array}{l}\text { Domínios } \\
\text { Cognitivos/Verbos }\end{array}$} & Atividades/verbos \\
\hline & \multirow{5}{*}{$\begin{array}{l}\text { Atividades } \\
\text { Verbos }\end{array}$} \\
\hline Categoria & \\
\hline Descrição & \\
\hline Definição & \\
\hline Verbos & \\
\hline
\end{tabular}

Figura 2 - Relacionamento Domínios Cognitivos/Verbos com Atividades/Verbos.

Na fase de implementação, foi desenvolvido um novo módulo para o AVA Moodle chamado de "Mapa de Dependência", e acrescido a versão anterior desta ferramenta pedagógica a opção "adicionar atividade" que era exigida a sua inserção para cada objetivo educacional definido pelo professor (Figura 3). 


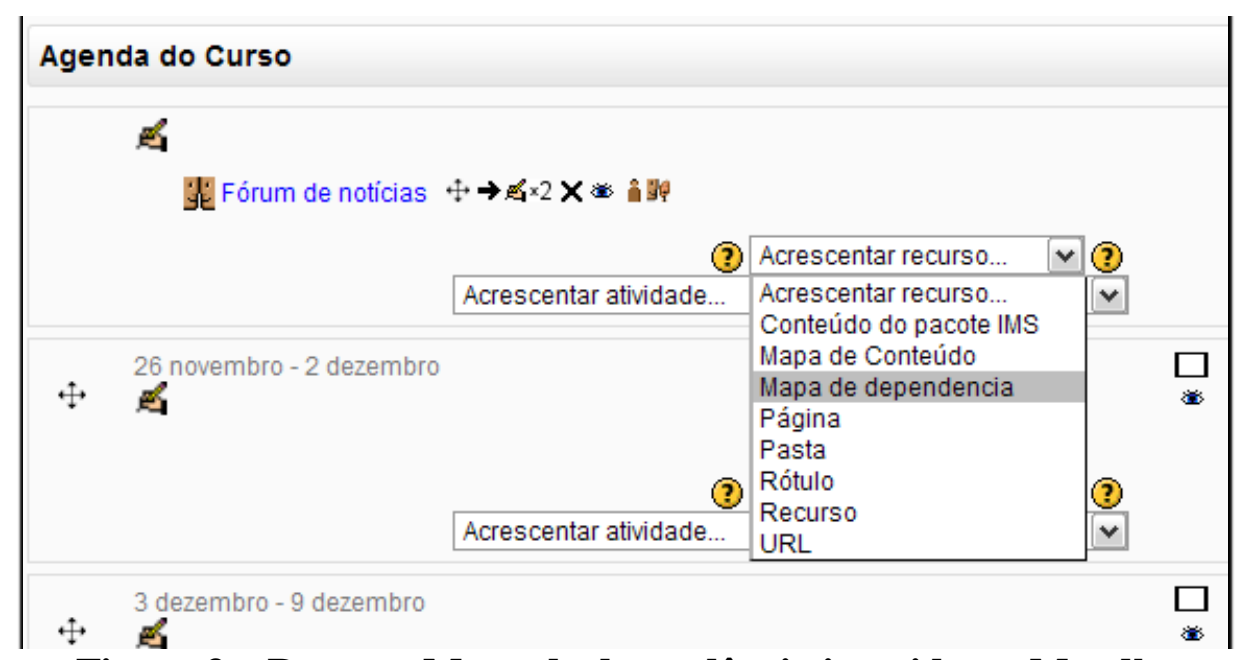

Figura 3 - Recurso Mapa de dependência inserido no Moodle.

Para os testes foi criada, através do AVA Moodle, uma turma de capacitação de professores através do curso de formação em Novas Tecnologias da Informação e Comunicação na Educação - NTICE (Figura 4) que foi desenvolvida no segundo semestre de 2012, com o objetivo de avaliar o desempenho dos alunos através da realização das atividades propostas durante o planejando do curso em relação aos objetivos educacionais definidos pelo professor no MD [Lima 2009].

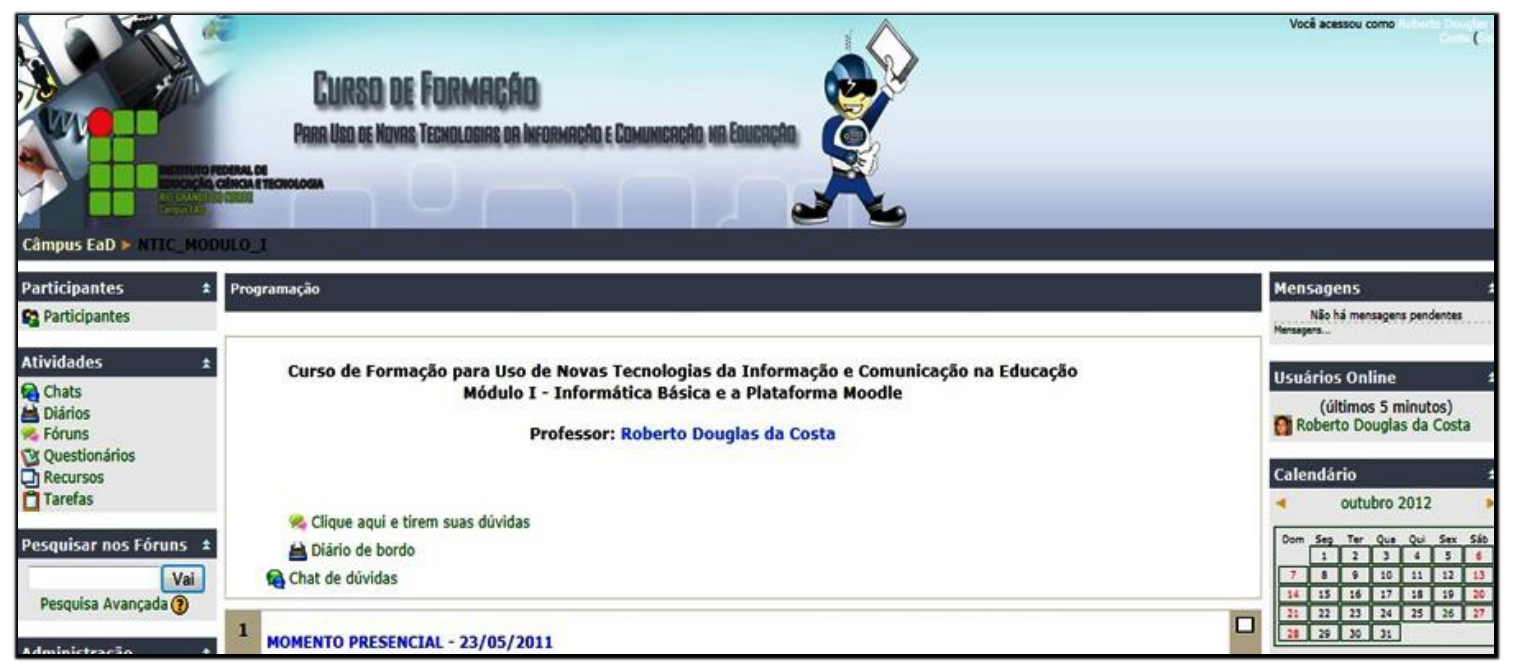

Figura 4 - Página do curso NTICE disponibilizada através do AVA Moodle.

E por último, foi feita uma pesquisa de avaliação entre os doze alunos e os dois professores do curso para saber se o uso da ferramenta MD, juntamente com o módulo de sugestão de atividades se ajudou ou não no direcionamento da aprendizagem do aluno.

Como resultado desta pesquisa, ficou constatado por parte dos professores que a definição dos objetivos educacionais relacionados às atividades avaliativas do AVA trabalhado, ajudou no esclarecimento do entendimento do aluno ao executar a atividade proposta. Desta maneira o professor pode entender o que o aluno estava compreendendo e se sua compreensão estava ou não conforme o objetivo educacional traçado.

Para os alunos ficou constatado que houve uma melhoria no acompanhamento dos estudos por parte dos professores proporcionado assim uma melhora no aprendizado, outro fator destacado foi uma maior agilidade no processo de feedback 
proporcionando assim, principalmente para os alunos que se encontravam em dificuldade, tempo hábil para retomar os estudos.

\section{Infraestrutura Utilizada}

Para este estudo foi criado um curso de capacitação em Novas Tecnologias para tutores e foi desenvolvido no Campus de Educação a Distância (EaD) do Instituto Federal de Educação, Ciência e Tecnologia do Rio Grande do Norte - IFRN, no qual já trabalhar com o desenvolvimento de cursos através do Moodle versão 1.9, no qual foi usado para desenvolver este referido projeto de pesquisa.

Segundo o site oficial do Moodle, este AVA é um pacote de softwares para produzir cursos baseados na Internet e Web sites. É um framework de construcionismo social da educação. Foi desenvolvido na linguagem de programação PHP, que é uma linguagem modulada, ideal para uso em servidores Web, é pode ser executado em qualquer computador que possua um Sistema de Gerenciamento de Banco de Dados (SGBD) compatível com SQL (Structured Query Language), sendo o MySQL o mais utilizado [Moodle 2013].

Pode ser executado sobre os sistemas operacionais Windows, Mac e várias distribuições Linux, isso lhe garante uma alta portabilidade, visto que é possível executá-lo nos sistemas operacionais de maior uso.

Acoplando a ele, usamos o bando de dados MySql que é um projeto de SGBD open-source e como servidor Web usamos o Apache. O plugin foi desenvolvido em PHP.

\section{Considerações Finais}

A avaliação é, e sempre será uma enorme preocupação no processo de ensinoaprendizado. É através dela que o docente e o discente colhem resultados de um aprendizado discorrido durante certo período de tempo descrito em um curso ou disciplina.

Esses resultados analisados podem ser apresentados de várias maneiras e utilizando alguns métodos avaliativos apresentados anteriormente, onde o mais tradicional é o método de avaliação somativa.

A utilizando do método de avaliação formativa, onde o acompanhamento evolutivo do aprendizado do aluno e muito importante, traz tanto para o professor como para o aluno uma maior transparência do processo de ensino-aprendizado, disponibilizando para o professor recursos para que ele possa alinhar o aprendizado do aluno, através de feedback adquirido ao final da execução das atividades dispostas pelos professores.

Por isso, a importância da inserção do módulo avaliação junto a ferramenta de planejamento acadêmico MD, que ira proporcionar ao professor um maior alinhamento como seus alunos e um maior acompanhamento do seu aprendizado.

Através da turma piloto onde o MD foi testado junto com módulo de avaliação acoplado, foi constatado que a ferramenta supriu a necessidade da comunidade docente que desenvolveu atividades neste curso, auxiliando-os no reconhecimento das verdadeiras deficiências dos alunos e trabalhando individualmente essas carências para suprir as necessidades de cada um deles, no que se diz respeito à assimilação dos conteúdos ministrado durante o transcorrer do curso ou disciplina. 


\section{Referências}

BLOOM, B. S.; ENGELHART, M. D.; FURST, E. J.; HILL, W. H.; KRATHWOHL, D. R. Taxonomia de objetivos educacionais - domínio cognitivo. Globo: Porto Alegre - RS. 1977.

COELHO NETO, J.; BERNARDELli, M. S.; PESSOA, M. P.; GUILHEM, S.; MALUCELLI, A.; REINEHR, S. O uso das TIC na formação de professores de escolas que obtiveram baixo IDEB. In: XXII Simpósio Brasileiro de Informática na Educação - SBIE. Aracajú - SE. p.988-996. 2011.

COSTA, R. D.; LIMA, R. W.; SILVA, T. R.; ROCHA, S. M. P. T.; FERNANDES, D. K. Análise quantitativa do uso das atividades avaliativas do Ambiente Virtual de Aprendizagem Moodle e sua classificação cognitiva segundo a taxonomia de Bloom. In: VI MoodleMoot Brasil, p. 7 - 16. São Paulo - SP. 2012.

FERRAZ, A. P. C. M.; BELHOT, R. V. Taxonomia de Bloom: revisão teórica e apresentação das adequações do instrumento para definição de objetivos instrucionais. Revista Gestão e Produção - Departamento de Engenharia de Produção - Universidade de São Carlos, V.17, nº 2, p. 421-431. 2010.

GLUZ, J. C.; GALAFASSI, C.; PENTEADO, F. Suporte Técnico/Pedagógico aos OAs pelos AVAs: uma Análise Comparativa das Pesquisas Recentes. In: XXII Simpósio Brasileiro de Informática na Educação - SBIE. Aracajú - SE. p. 294-303. 2011.

LIMA, R. W.; FIALHO, S. V. Mapa de Dependências: uma metodologia para o planejamento e avaliação da aprendizagem. In: International Conference on Engineering and Technology Education, 2008, Peruíbe/Santos-SP. X International Conference on Engineering and Technology Education, 2008.

LIMA, R. W. Mapa de Conteúdos e Mapa de Dependências: Ferramentas Pedagógicas para uma Metodologia de Planejamento baseada em Objetivos Educacionais e Sua Implementação em um Ambiente Virtual de Aprendizagem. Tese de Doutorado - UFRN, 2009.

LOMENA, M. Benjamin Bloom. 2006. Disponível em: <http://www.everything2.com/ index.pl?node_id=143987>. Acesso em: nov. 2012.

MOODLE. Modular Object-Oriented Dynamic Learning Environment. 2013. Disponível em <http://moodle.org> . Acesso em: nov. de 2013.

PIVA JÚNIOR, D.; PUPO, J. R. S.; GAMEZ, L. EAD na prática: planejamento, métodos e ambientes de educação online. Rio de Janeiro: Elsevier. 2011.

SILVA, R. S. Moodle para autores e tutores. São Paulo: Novatec, 2011.

SILVA, T. R.; LIMA, R. W.; MESQUITA, H. H. O.; MARQUES, C. K. M.; COSTA, R. D; ROCHA, S. M. P. T.; MEDEIROS, R. A. OBA-MC: um modelo de Objeto de Aprendizagem centrado no processo de ensino-aprendizagem utilizando o padrão SCORM. In: XXIII Simpósio Brasileiro de Informática na Educação - SBIE. Rio de Janeiro- RJ, 2012. 